\title{
PIRY VIRUS ANTIBODIES IN INHABITANTS OF RIO DE JANEIRO
}

\author{
MARCIA G. CASTRO; AMÉLIA P. A. TRAVASSOS DA ROSA*; \\ RICARDO LOURENÇO-DE-OLIVEIRA; RITA M. R. NOGUEIRA*; HERMANN G. \\ SCHATZMAYR* \& LEONIDAS M. DEANE
}

Departamento de Entomologia *Departamento de Virologia, Instituto Oswaido Cruz, Av. Brasil 4365, 21045-900 Rio de Janeiro, RJ, Brasil **Instituto Evandro Chagas, Av. Almirante Barroso, 492, 66090-000 Belém, PA, Brasil

Serological surveys carried out in Brazil and other South American countries have indicated that human infections by Piry virus (Rhabdoviridae, Vesiculovirus) are limitated to some areas of Brazil. Neutralizing antibodies against this virus has been detected in 4 to $17 \%$ of residents in communities surveyed in the Amazon basin. Antibody prevalence rates of $20.3 \%$ have been found among immigrants from southern Brazil (F. P. Pinheiro et al., 1974, PAHO Bull., 8: 111-122); $14.3 \%$ of adult residents in the southeastern region, Ribeirão Preto, São Paulo state were immune (L. T. M. Figueiredo et al., 1985, Rev. Inst. Med. Trop., S. Paulo, 27: 157-161); $8.0 \%$ of blood donors in Uberaba, Minas Gerais state, had neutralizing antibodies (J. Tavares Neto et al., 1990, Rev. Inst. Med. Trop., S. Paulo, 32: 211-214) whereas $13-33 \%$ of residents in the northeastern region, Bahia state, appeared to be immune to the agent (J. Tavares Neto, personal communication).

In order to verify the occurrence of antibodies against Piry virus in inhabitants from the city of Rio de Janeiro, Rio de Janeiro state, 389 sera were screened by neutralization test, utilizing IC inoculation of two to four day old mice. The constant serum technique (final dilution 1:8) was used with 10 fold varying virus dilutions, according to R. E. Shope \& G. E. Sather (1979, Arboviruses, p. 766-814. In E. H. Lennette \& N. J. Schimidt, (eds), Diagnostic procedures for viral rickettsial and clamydial infections. Amer. Publ. Hlth. Assoc., Washington). Individuals were considered positive when the serum titers were equal to or higher than $1.7 \mathrm{log}$ for neutralization index. Blood was taken by vein puncture mainly in patients at a hospital in Vargem Grande, but a few

Received 25 February 1993.

Accepted 10 August 1993. others were also collected at the dwellings, in Granjas Calábria and Vargem Grande, both in Jacarepaguá county. These localities include peri-urban and nural areas in the west border of the city of Rio de Janeiro and were chosen for being sites of mosquito collections in an arbovirus survey (M. G. Castro et al., 1991, Virológica 91, abstracts, p. 4). Personal data from the group was obtained, including previous living places.

Blood samples were collected in 1990 and 1991 and sera were stored at $-20^{\circ} \mathrm{C}$ until they could be tested for Piry antibody.

Neutralizing antibodies for Piry virus were found in 69 out of 389 persons between 7 to 85 years old (Table). The highest rates of positivity was found among females, $19.5 \%$ against $14.4 \%$ in males and people older than $30(84.1 \%)$ as shown at Table and Figures. Only 2 children, 7 and 12 years old had antibodies to Piry virus.

The earlier arbovirus antibody surveys performed in Rio de Janeiro (M. Bruno-Lobo et al., 1961, An. Microbiol., 9: 155-191; F. P. Pinheiro et al., 1975, Intervirology, 5: 93-96), the last one using neutralizing antibodies, showed immunity to St. Louis encephalitis, Yellow fever, Ilhéus and Bussuquara viruses in the same area. The present paper is the first detection of Piry virus neutralizing antibodies in Rio de Janeiro, including 17 out of 121 persons examined, which were born and always lived in the area (Table). This percentage of $14 \%$ is similar to those reported in the neighbouring states of S. Paulo and Minas Gerais (L. T. M. Figueredo et al.; J. Tavares Neto et al., loc. cit.).

Although 45 autochthonous children less than nine years old have been tested, only one was positive, suggesting low circulation of Piry 
virus in the last years; the other positive people were fairly distributed by age and the presence of 4 persons more than 60 years old, indicate that Piry virus must be present since long time in the area (Figs 1, 2).

The antibodies found in the population which has lived in other parts of the country, where they may have been infected, pointed out for the presence of Piry virus in different parts of Brazil.
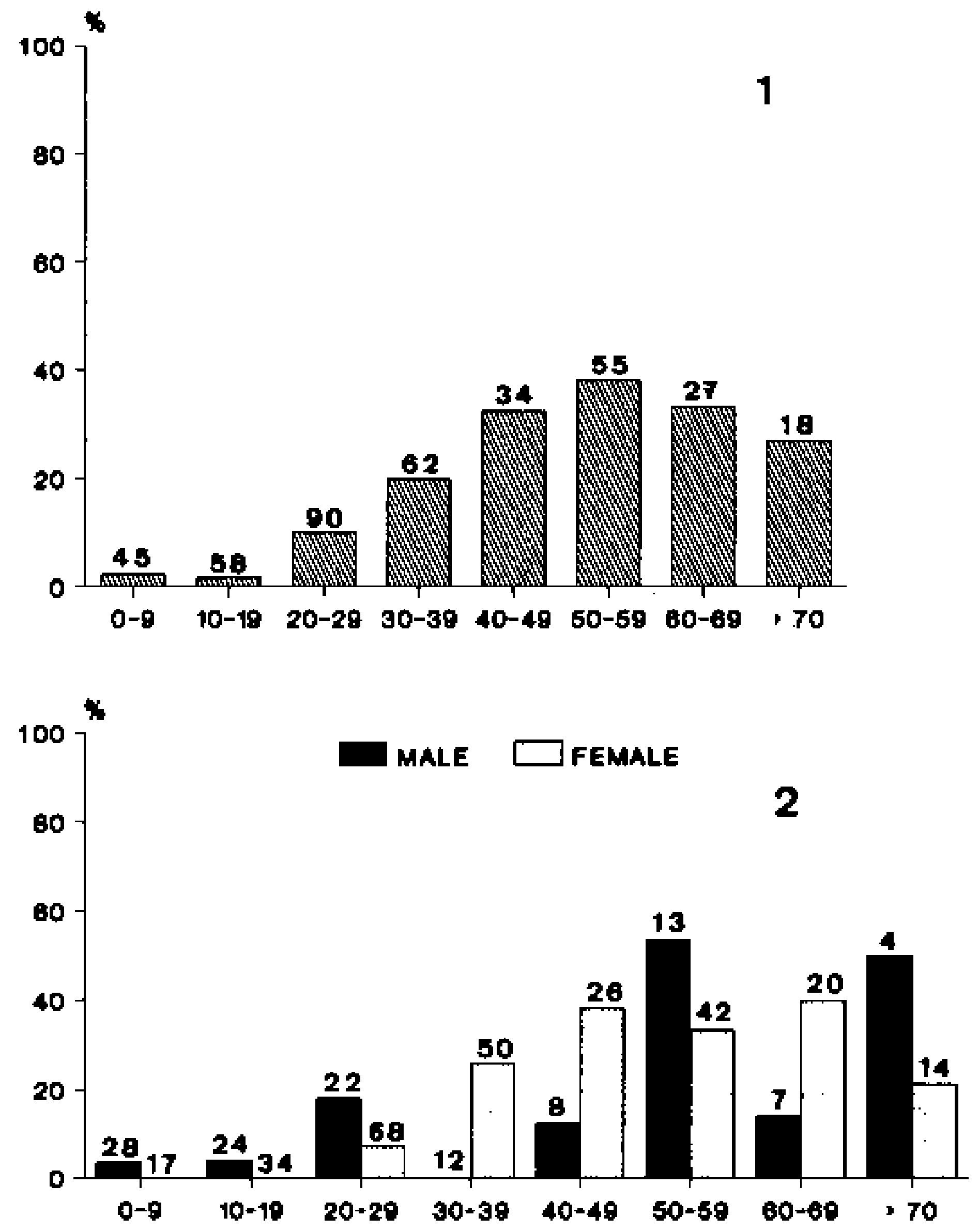

Figs 1, 2: percentage of positive individuals for Piry virus neutralizing antibodies living in Jacarepaguá, Rio de Janeiro, in both sexes, and by sex and age, respectively; the number of individuals tested is shown at the top of the bars.

\section{TABLE}

Neutralization antibodies titers to Piry virus in sera of positive individuals living in different localities in the county of Jacarepaguá, Rio de Janeiro

\begin{tabular}{lllllr}
\hline Person & Age & Sex & Profession & Locality & Result: ILN \\
\hline A.C.C. & 72 & $\mathrm{~F}$ & Housemaid & Estrada Boca do Mato & $<5.0 ;>3.2$ \\
A.C.O. & 58 & $\mathrm{~F}$ & Housewife & Granjas Calabria & $>4.7$ \\
A.C.S. & 46 & $\mathrm{M}$ & & Granjas Calabria & $>2.7$ \\
A.D.M. & 27 & $\mathrm{~F}$ & Comercializer & Vargem Grande & $<4.8 ;>3.4$ \\
A.G. & 26 & $\mathrm{M}$ & & Rio Bonito & $<5.2 ;>2.8$ \\
A.G.R. & 70 & $\mathrm{~F}$ & & Vargem Grande & $<4.8 ;>3.2$ \\
A.L.S. & 59 & $\mathrm{~F}$ & Housemaid & Recreio & $<4.5 ;>3.4$ \\
A.M.S. & 30 & $\mathrm{~F}$ & Civil servant & Vargem Grande & $<4.5 ;>3.5$ \\
A.P.C. & 69 & $\mathrm{~F}$ & Housemaid & Vargem Grande & $<4.5 ;>4.0$ \\
A.P.N. & 65 & $\mathrm{~F}$ & Housemaid & Estrada dos Bandeirantes & $<4.6 ;>3.9$ \\
A.R.S. & 51 & $\mathrm{M}$ & & Recreio & $<4.5 ;>4.0$ \\
A.S.S. & 58 & $\mathrm{M}$ & Landman & Granjas Calabria & $>2.7$ \\
B.J.P. & 85 & $\mathrm{M}$ & & Caminho do Portello & $>3.1$ \\
C.C. & 68 & $\mathrm{~F}$ & Housemaid & Vargem Grande & $<4.5 ;>3.6$ \\
C.C.S ${ }^{a}$ & 68 & $\mathrm{~F}$ & Housemaid & Vargem Grande & $<4.5 ;>4.0$ \\
C.V.N.L. & 26 & $\mathrm{~F}$ & & Recreio & $<4.5 ;>4.0$ \\
D.A. & 53 & $\mathrm{~F}$ & Housemaid & Estrada dos Bandeirantes & $<4.8 ;>3.6$ \\
D.D.S. & 41 & $\mathrm{~F}$ & Housemaid & Vargem Grande & $<4.8 ;>3.7$ \\
D.F. & 58 & $\mathrm{M}$ & & Vargem Pequena & $<4.5 ;>3.6$ \\
D.G.D. & 51 & $\mathrm{~F}$ & Housemaid & Vargem Grande & $<4.5 ;>3.6$ \\
E.R.S. & 66 & $\mathrm{~F}$ & Housewife & Taquara & $<4.5 ;>4.0$ \\
E.T.S. & 23 & $\mathrm{~F}$ & & Recreio & $<5.0 ;>3.2$ \\
G.M. & 49 & $\mathrm{~F}$ & Housewife & & $<4.6 ;>3.6$ \\
G.S.L. & 31 & $\mathrm{~F}$ & & Curicica & $<4.5 ;>3.4$
\end{tabular}




\begin{tabular}{|c|c|c|c|c|c|}
\hline I.F.O. & 55 & $\mathbf{F}$ & Woman servant & Recreio & $<5.0 ;>3.4$ \\
\hline I.M.J. & 41 & $\mathbf{F}$ & & Taquara & $<4.5 ;>4.0$ \\
\hline J.A.E. & 28 & $\mathbf{M}$ & & Vargem Pequena & $<4.5 ;>3.9$ \\
\hline J.A.S. & 55 & $\mathbf{F}$ & Housemaid & Vargem Grande & $>2.7$ \\
\hline J.B.O. & 59 & $\mathrm{~F}$ & Housemaid & Vargem Grande & $<4.5 ;>3.6$ \\
\hline J.M.G. ${ }^{a}$ & 71 & $\mathbf{M}$ & Carpenter & Benvindo Novaes & $>4.7$ \\
\hline J.R.M. & 29 & $\mathbf{M}$ & & Vargem Grande & $>4.4$ \\
\hline J.R.S. & 26 & $\mathrm{M}$ & & Estrada dos Bandeirantes & $<5.0 ;>3.1$ \\
\hline L.F.A.O. ${ }^{a}$ & 7 & M & Student & Vargem Grande & $<4.5 ;>4.0$ \\
\hline L.F.M. & 35 & $\mathrm{~F}$ & & Recreio & $<4.5 ;>3.6$ \\
\hline L.G.L. ${ }^{a}$ & 54 & $\mathrm{M}$ & & Benvindo Novaes & $>3.0$ \\
\hline L.H.S. & 27 & $\mathbf{F}$ & & Restinga & $<4.5 ;>3.7$ \\
\hline L.M.L. ${ }^{a}$ & 53 & $\mathrm{~F}$ & Housewife & Benvindo Novaes & $>3.8$ \\
\hline L.V.V. & 49 & $\mathrm{~F}$ & & Vargem Pequena & $<4.5 ;>3.4$ \\
\hline M.A.F.D. & 26 & $\mathbf{F}$ & & Rio Bonito & $<4.5 ;>3.9$ \\
\hline M.A.G. & 35 & $\mathrm{~F}$ & Housewife & Vargem Grande & $<5.5 ;>2.6$ \\
\hline M.A.S.F. ${ }^{a}$ & 32 & $\mathrm{~F}$ & Housewife & Granjas Calabria & $>2.6$ \\
\hline M.D.F ${ }^{a}$ & 50 & $\mathrm{~F}$ & Housewife & Benvindo Novaes & $>4.8$ \\
\hline M.F.C. & 70 & $\mathrm{~F}$ & Housermaid & Estrada dos Bandeirantes & $<4.5 ;>3.7$ \\
\hline M.G.M. ${ }^{a}$ & 34 & $\mathrm{~F}$ & Housewife & Recreio & $<5.0 ;>3.5$ \\
\hline M.G.P. & 54 & $\mathrm{~F}$ & & Recreio & $<4.6 ;>3.9$ \\
\hline M.G.S. ${ }^{a}$ & 46 & $\mathbf{F}$ & Housewife & Granjas Calabria & $>2.6$ \\
\hline M.G.S. & 61 & $\mathrm{~F}$ & & Vargem Grande & $<4.5 ;>3.6$ \\
\hline M.G.S. ${ }^{a}$ & 43 & $\mathrm{~F}$ & & Barra da Tijuca & $<5.8 ;>2.7$ \\
\hline M.J.D. & 69 & $F$ & & Taquara & $<4.5 ;>4.0$ \\
\hline M.J.S.L. & 49 & $\mathrm{~F}$ & Housemaid & Vargem Grande & $<4.5 ;>4.0$ \\
\hline M.L.B.O. & 34 & $\mathrm{~F}$ & Housemaid & Rio Morto & $<4.5 ;>3.3$ \\
\hline M.L.S. & 54 & $\mathrm{~F}$ & Housemaid & Rio Bonito & $<4.5 ;>3.7$ \\
\hline M.N.S. & 32 & $\mathrm{~F}$ & & Vargem Pequena & $<4.5 ;>3.7$ \\
\hline M.S.M. & 34 & $\mathrm{~F}$ & & Vargem Grande & $<4.6 ;>3.4$ \\
\hline O.L.T. ${ }^{a}$ & 55 & $\mathbf{M}$ & Tiller & Caminho do Portello & $>3.3$ \\
\hline R.I. ${ }^{a}$ & 32 & $\mathbf{F}$ & Civil servant & Rio Bonito & $<4.5 ;>3.3$ \\
\hline R.S. & 12 & $\mathbf{M}$ & Studant & Barra da Tijuca & $<5.6 ;>3.1$ \\
\hline R.S.A. & 60 & $\mathrm{~F}$ & Housemaid & Vargem Grande & $<4.5 ;>4.0$ \\
\hline R.V. & 67 & $\mathbf{M}$ & & Campo Grande & $<4.5 ;>3.5$ \\
\hline S.C.J. & 59 & $\mathrm{M}$ & Gardener & Rua Pedro Afonso & $<4.5 ;>3.9$ \\
\hline S.D.M.A. & 40 & $\mathbf{F}$ & Housemaid & Barra da Tijuca & $<4.5 ;>3.7$ \\
\hline S.D.O. & 43 & $\mathrm{~F}$ & Housemaid & Vargem Grande & $<4.5 ;>3.7$ \\
\hline S.M.S. & 31 & $\mathbf{F}$ & & Camorim & $<4.5 ;>4.3$ \\
\hline S.P.D. & 34 & $F$ & & Taquara & $<4.8 ;>3.7$ \\
\hline S.R.S. & 50 & $M$ & Landman & Granjas Calabria & $>4.2$ \\
\hline V.G.S. & 52 & $\mathrm{M}$ & Man servant & Vargem Grande & $<6.2 ;>2.5$ \\
\hline V.M.C. & 34 & $\mathrm{~F}$ & & Av, das Americas & $<4.5 ;>3.7$ \\
\hline Z.A.S. ${ }^{a}$ & 28 & $\mathrm{~F}$ & & Vargem Grande & $<4.6 ;>4.2$ \\
\hline Z.R.R. ${ }^{a}$ & 52 & $\mathrm{~F}$ & Housemaid & Vargem Grande & $<4.5 ;>4.3$ \\
\hline
\end{tabular}

$a$ : individuals that were born and have always lived in Rio de Janeiro state. 